DFUB $6 / 2003$

\title{
Monte Carlo simulation of an experiment looking for radiative solar neutrino decays
}

\author{
S. Cecchini ${ }^{\mathrm{a}, \mathrm{b}}$ D. Centomo ${ }^{\mathrm{a}}$ G. Giacomelli ${ }^{\mathrm{a}}$ V. Popa ${ }^{\mathrm{a}, \mathrm{c}}$ \\ C.G. Şerbănuţ, a,c \\ a Dipartimento di Fisica dell'Università and INFN Sezione di Bologna, I-40127 \\ Bologna, Italy \\ ${ }^{\mathrm{b}} I A S F / C N R, I-40129$ Bologna, Italy \\ ${ }^{\mathrm{c}}$ Institute for Space Sciences, R-77125 Bucharest Măgurele, Romania
}

\begin{abstract}
We analyse the possibility of detecting visible photons from a hypothetical radiative decay of solar neutrinos. Our study is focused on the simulation of such measurements during total solar eclipses and it is based on the BP2000 Standard Solar Model and on the most recent experimental information concerning the neutrino properties. Our calculations yield the probabilities of the decays, the shapes of the visible signals and the spectral distributions of the expected photons, under the assumption that solar neutrino oscillations occur according to the LMA model.
\end{abstract}

Key words: Solar neutrinos, Decays of heavy neutrinos, Neutrino mass and mixing, Total solar eclipses, Numerical simulations

PACS: 96.60.Vg, 13.35.Hb, 14.60.Pq, 95.85.Ry, 02.60.Cb

\section{Introduction}

In the last few years it has become clear that neutrinos have non-vanishing masses, and that the neutrino flavor eigenstates $\left(\nu_{e}, \nu_{\mu}\right.$ and $\left.\nu_{\tau}\right)$ are superpositions of mass eigenstates $\left(\nu_{1}, \nu_{2}\right.$ and $\left.\nu_{3}\right)$. For a recent review, see [1]. In this context, neutrinos could undergo radiative decays, e.g. $\nu_{2} \rightarrow \nu_{1}+\gamma$, as initially suggested in [2]. The present status of decaying theory is sumarized in [3]. Such decays request that the involved neutrinos have a non-vanishing electric dipole moment; the very stringent existing experimental limits refer to the flavor neutrino eigenstates and they are not directly applicable to possible dipole moments of mass neutrino eigenstates. 
In a pioneering experiment performed during the Total Solar Eclipse (TSE) of October 24, 1995 a search was made for visible photons emitted through possible radiative decays of solar neutrinos during their flight between the Moon and the Earth [4]. In the analysis of the data, the authors assumed that all neutrinos have masses of the order of few eV, $\Delta m_{12}^{2}=m_{2}^{2}-m_{1}^{2} \simeq 10^{-5}$ $\mathrm{eV}^{2}$, and an average neutrino energy of $860 \mathrm{keV}$; furthermore they assumed that all decays would lead to visible photons, which would travel nearly in the same direction as the parent neutrinos, thus leading to a narrow spot of light coming from the direction of the center of the dark disk of the Moon.

Subsequently, Frère and Monderen made more accurate calculations on the shape of the expected signal [5], considering the Sun as an extended source of electron neutrinos with typical energies of the order of $1 \mathrm{MeV}$, a Lagrangian formalism for the radiative decay, different neutrino masses $(1 \mathrm{eV}$ or $0.5 \mathrm{eV})$, and mass squared differences $\Delta m^{2}$ of $10^{-5}, 0.25$ and $1 \mathrm{eV}^{2}$; they have shown that the expected signal could have an extended angular pattern.

Some of the authors of this paper were involved in two experiments along the line of [4], during the total solar eclipses of August 11, 1999 (in Romania) $[6,7,8]$, and of June 21, 2001 (in Zambia) [8]. In 1999 the bad weather conditions did not allow the planned observations, but we could use a videotape filmed by a local television (Râmnicu Vâlcea). The analysis of the data was performed in the hypothesis of a possible

$$
\nu_{2} \rightarrow \nu_{1}+\gamma
$$

decay, with $m_{2}>m_{1}$. For the analysis of the 1999 data we have chosen the two $\Delta m^{2}$ values suggested by the MSW SMA (Small Mixing Angle) and LMA (Large Mixing Angle) solutions of the Solar Neutrino Problem (SNP), allowed by the then available experimental data from solar neutrino experiments. We developed a Monte Carlo (MC) simulation of the radiative solar neutrino decay, considering the solar neutrino energy spectrum predicted by the Standard Solar Model (SSM) [9] and the mass of the $\nu_{1}$ in the range of $1-10 \mathrm{eV}$, as it was expected at that time. Since the angular resolution of the data was not very good, we considered the Sun as a pointlike neutrino surce. The simulation has shown that the expected signal should be a narrow spot of light in the direction of the center of the Sun, and allowed an evaluation of the fraction of decays yielding visible photons as function of the chosen neutrino $\nu_{1}$ mass $m_{1}$ and $\Delta m^{2}$ values.

The 2001 experiment lead to better quality data, so the real spatial distribution of the solar neutrino yield had to be considered. Furthermore, the recent SNO results $[10,11]$ favour the LMA solution and could indicate also the presence of a $\nu_{\tau}$ component in the solar neutrino flux at the Earth level. 
The WMAP (Wilkinson Microwave Anisotropy Probe) results after the first year of flight [12] limit the sum of the masses of the three neutrino species to $0.23 \mathrm{eV}$ ( $95 \%$ Confidence Level).

In this paper we present a more complete simulation. We assume that $m_{1}<$ $m_{2}<m_{3}$ where $m_{1}, m_{2}, m_{3}$ are the masses of the $\nu_{1}, \nu_{2}$ and $\nu_{3}$ mass eigenstates, respectively; but we restrict our analysis to a two generation mixing scenario, assuming the present mass differences obtained from solar neutrino experiments, the LMA solution with $\Delta m_{12}^{2}=6 \times 10^{-5} \mathrm{eV}^{2}$. Since SNO suggests also the presence of $\nu_{3}$ in the solar neutrino flux, we considered also the mass difference measured by atmospheric neutrino experiments $[13,14,15]$ : $\Delta m_{13}^{2} \simeq \Delta m_{23}^{2}=2.5 \times 10^{-3} \mathrm{eV}^{2}$. We included in our MC all the details of neutrino production in the Sun, as given by the "BP2000" SSM [16].

The aim of our simulation is to give information on the shape of a possible decay signal of solar neutrinos (angular and energy distributions of the emitted photons) and to estimate the probabilities for the decay and the geometrical detection efficiency. This information should allow to obtain limits on the neutrino lifetimes from the experimental observations. As it is shown in the following sections, some of the input parameters of the code refer to the characteristics of a specific experiment. The results presented in this paper are obtained in the conditions of the observations made in 2001 in Zambia [8] with a digital videocamera.

\section{The Monte Carlo simulation}

The assumed geometry for the simulation of the radiative decay of solar neutrinos during a TSE is shown in Fig. 1. The notations in the figure will be used in the following subsections.

\subsection{Neutrino production inside the Sun}

In simulating the solar neutrino production, we used the "BP2000" SSM [16] in its numerical form directly available from [17]. The first step consists in chosing a specific reaction/decay yielding neutrinos (both from the p-p and the CNO cycles) according to its predicted contribution to the solar neutrino flux at the Earth. The neutrino energy and the radius $R$ of its production point are then randomly generated according to the SSM. In Fig. 2 we present the neutrino solar energy spectrum obtained from $3 \times 10^{7}$ generated neutrinos, and the distribution of the distance $R$ from the center of the Sun of the production points. 


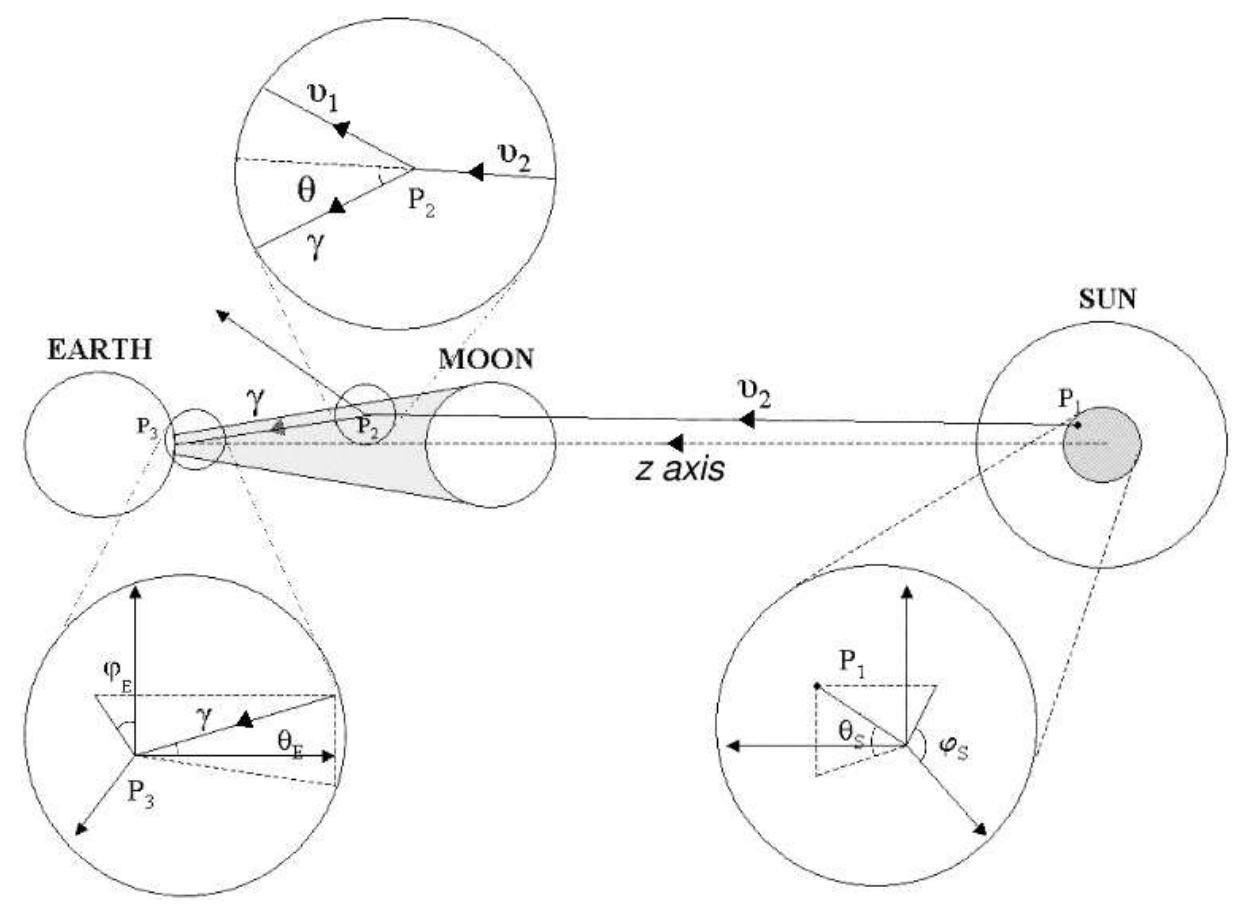

Fig. 1. A sketch of the geometry of the production of solar neutrinos, their possible radiative decay (in the space between the Moon and the Earth) and the detection of the emitted photon, during a TSE. The $z$ axis is directed from the center of the Sun to the observation point on the Earth (or center of the Earth)
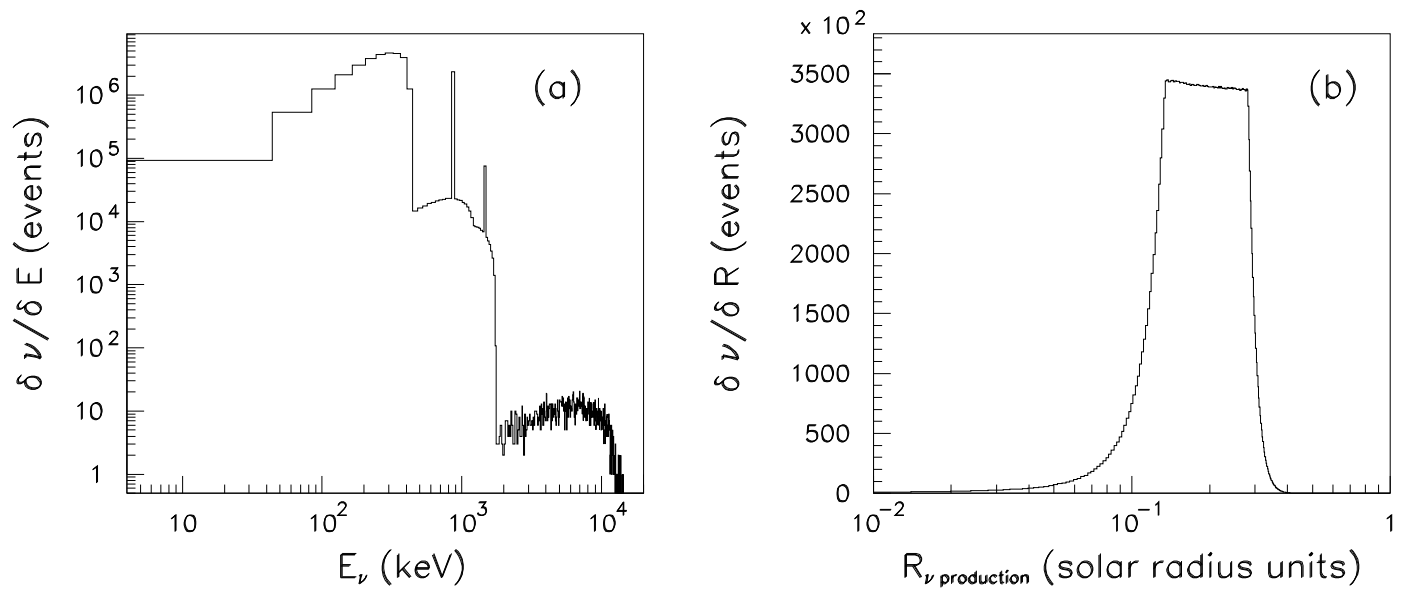

Fig. 2. Monte Carlo simulations according to the "BP2000" SSM. (a)The energy spectrum of solar neutrinos; the ${ }^{8} B$ neutrinos are the few neutrinos with energy between $10^{3}$ and $10^{4} \mathrm{keV}$. (b) The distribution of the radial distance of the production points from the center of the Sun.

In order to ensure a uniform distribution of the birth points of the neutrinos ( $P_{1}$ in Fig. 1) on each shell $\Delta R$ at radial distance $R$, we determine for each neutrino the angular spherical coordinates generating uniformely the cosinus of the zenithal angle $\left(\theta_{S}\right.$ in Fig. 1, assuming the " $\mathrm{z}$ " axis oriented from the centre of the Sun towards the centre of the Earth) and the polar angle $\left(\phi_{S}\right.$ in 
Fig. 1.).

\subsection{Propagation of solar neutrinos and of the decay photons}

The solar neutrinos are produced as $\nu_{e}$ 's, thus as a superposition of neutrino mass states. After leaving the Sun, during their flight in the interplanetary space, their weak flavor is irrelevant.

In our simulation we consider the $\nu_{2}$ (or $\nu_{3}$ ) component of the neutrino flux, that may decay into the lower mass state $\nu_{1}$ and a photon. As we are interested in the possibility to observe such photons during a total solar eclipse, we impose that the decay processes take place in the space between the Moon and the Earth, inside the shadow cone of the Moon (otherwise the separation of the decay photons from the solar light backround would be impossible). Furthermore, the decay photons must reach the detector (a CCD-equipped telescope, a digital camera or some other observation systems of the same kind).

The next step in the simulation is to define the incidence direction of the decay photon on the detector, situated on the Earth surface at the location $P_{3}$ (see Fig. 1). We generate uniformly the cosinus value of the "local" zenith angle $\theta_{E}$ inside the shadow cone (or in the cone subtended by the telescope or by the analysis apperture if smaller) and then the value of the "local" azimuthal angle $\phi_{E}$, as defined in Fig. 1.

The probability of neutrino radiative decays (thus of their lifetime) depends on their electric dipole momentum, which, for the weak flavor eigenstates, is limited by the existing experimental data [18]. Those limits are not directly applicable for the mass eigenstates. We may assume that the lifetime of $\nu_{2}$ is much larger than the time of flight from the Sun to the Earth (otherwise the experiment would be impossible). This implies that the decay points of the massive solar neutrinos are uniformely distributed along their path from the Moon to the Earth.

As we already defined the direction of the "detected" photon, we cannot choose the decay point of the neutrino using the above assumption; instead we take advantage of the nearly cylindrical symmetry of our problem to choose the decay point (point $P_{3}$ in Fig. 1) randomly along the photon path and then determine the neutrino path as the segment starting in $P_{1}$ and ending in $P_{3}$. This does not affect the expected uniformity of the distribution of the decay points along the neutrino path from the Moon to the Earth, as shown in Fig. 3a. We also checked the isotropy on the incidence of solar neutrinos on the Moon surface (see Fig. 3b). 
(a)

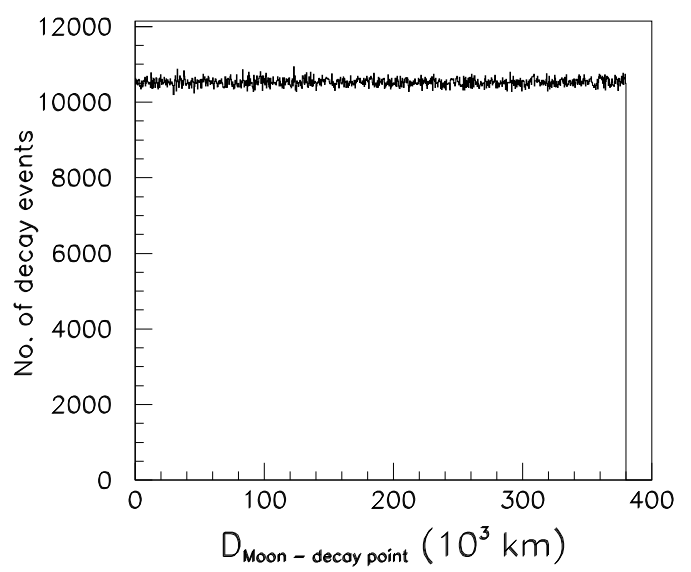

(b)

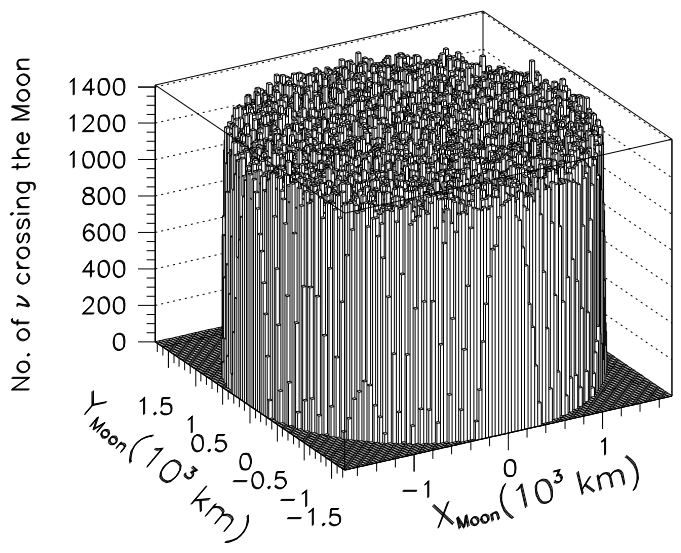

Fig. 3. "Isotropy" tests for the simulated solar neutrino flux: a) The distribution of neutrino path lengths from the Moon till their decay point. b) The distribution of the points of incidence of the solar $\nu$ 's on the Moon disk. One may notice that both distributions are uniform

\subsection{Neutrino decays}

For every simulated event we know (from the steps described in the above subsections) the 4-vector of the "heavy" neutrino $\nu_{2}$, its birth and decay points, and the direction of the photon emission; from the 4-momentum conservation we obtain the energy (in the laboratory reference frame, LRF) of the emitted $\gamma$

$$
E_{\gamma}=\frac{\Delta m^{2}}{2} \frac{1}{E_{\nu}-p_{\nu} \cos \theta}
$$

where $E_{\nu}$ and $p_{\nu}$ are the energy and the momentum of the parent $\nu_{2}$ neutrino, respectively, and $\theta$ is the emission angle of the photon relative to the direction of the $\nu_{2}$ momentum in the LRF (see Fig. 1).

In the center of mass $(\mathrm{CM})$ of the decaying $\nu_{2}$ neutrino, the probability density of the emission of a photon at the zenithal angle $\theta^{*}$ is given by:

$$
\frac{d \Gamma}{d\left(\cos \theta^{*}\right)}=K\left(1+\alpha \cos \theta^{*}\right)
$$

where the $\alpha$ parameter depends on the polarization state of the initial $\nu_{2}$ flux: $\alpha=0$ for unpolarized (Majorana) neutrinos, and $\alpha=\mp 1$ for left and right handed (Dirac) $\nu_{2}$ 's, respectively. The constant $K$ comes from the general description of the decay of a fermion into a fermion and a boson

$$
K=\frac{\alpha_{e}^{2}}{\pi^{2}} \frac{m_{2}}{\left(\Delta m^{2}\right)^{3}}\left(m_{1}^{2}+m_{2}^{2}+m_{1} m_{2}\right),
$$




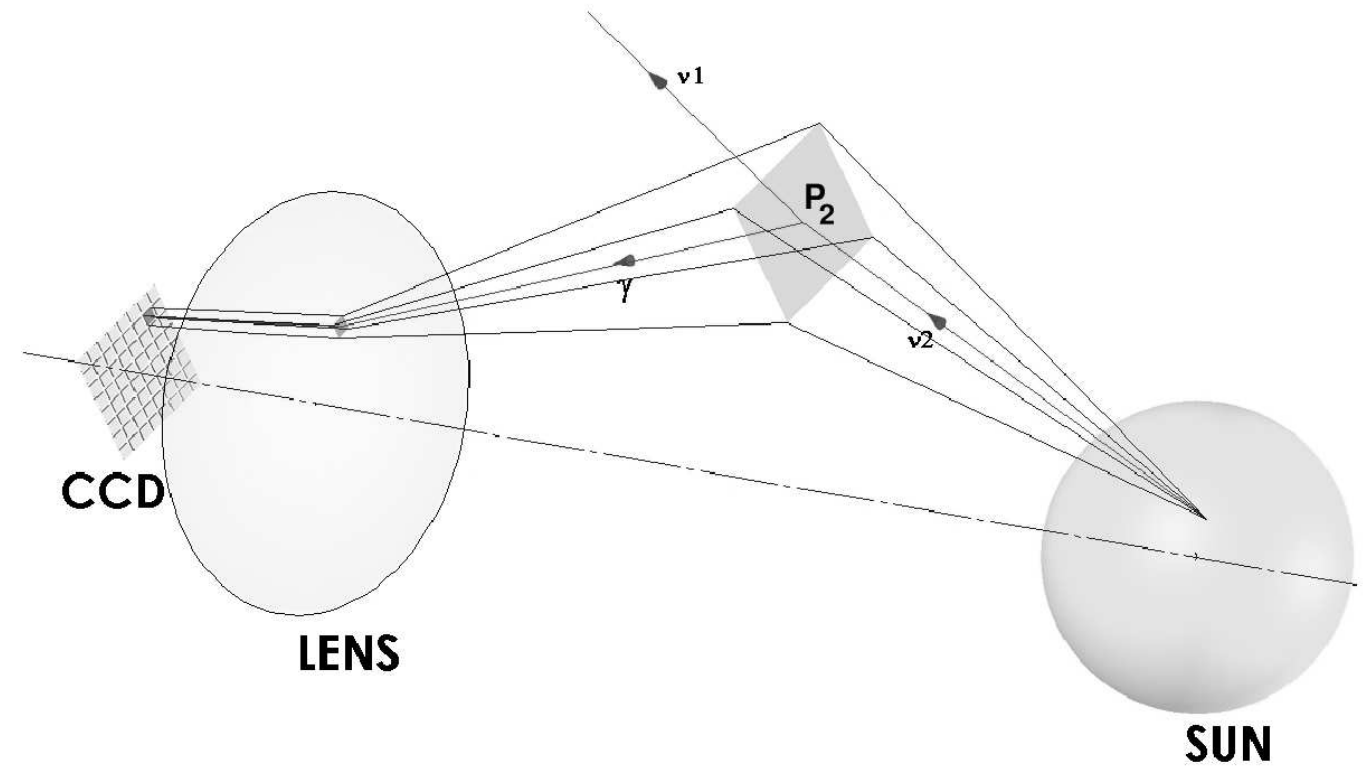

Fig. 4. A sketch showing the integration area for the probability density in Eq. 3. Any decay event inside the dark square around point $P_{2}$ would lead to a signal in the same CCD pixel. (In this sketch the Moon is not represented.)

where $\alpha_{e}$ is the fine structure constant.

In order to estimate the probability of each simulated event, one would have to integrate Eq. 3 inside the solid angle under which the emitted photon "sees" the detector. This is unpractical since the physical dimension of any usable detector is too small compared to the distance scales involved in the simulation. An equivalent approach is based on the observation that any imaging system (such as a CCD in the focal plane of some optical system) has a limited angular resolution, as the images of all point sources inside the solid angle covered by a single pixel will be superimposed. We can then numerically integrate Eq. 3 on the area around the decay point $P_{2}$ (see Fig. 4 for a sketch; the integration area is represented as the dark square centered in $P_{2}$ ) that corresponds to the angular aperture of a single pixel. The probabilities obtained in this way are used as weights for the MC generated events.

\section{Results and discussions}

The results presented in this Section are obtained assuming an angular resolution of each pixel of 10", and an angular aperture of the analysis (the maximum value of $\theta_{E}$ in Fig. 1) of 480", as in the case of the digital videocamera used during the 2001 TSE [8]. The mass $m_{1}$ of the $\nu_{1}$ mass eigenstate was considered in a range between $10^{-3} \mathrm{eV}$ and $0.3 \mathrm{eV}$; MC simulations were made for $\Delta m^{2}=6 \times 10^{-5} \mathrm{eV}^{2}$ and also for $\Delta m^{2}=2.5 \times 10^{-3} \mathrm{eV}^{2}$. For the polarization parameter $\alpha$ in Eq. 3, we considered three possible values: $-1,0$ and 1 . 


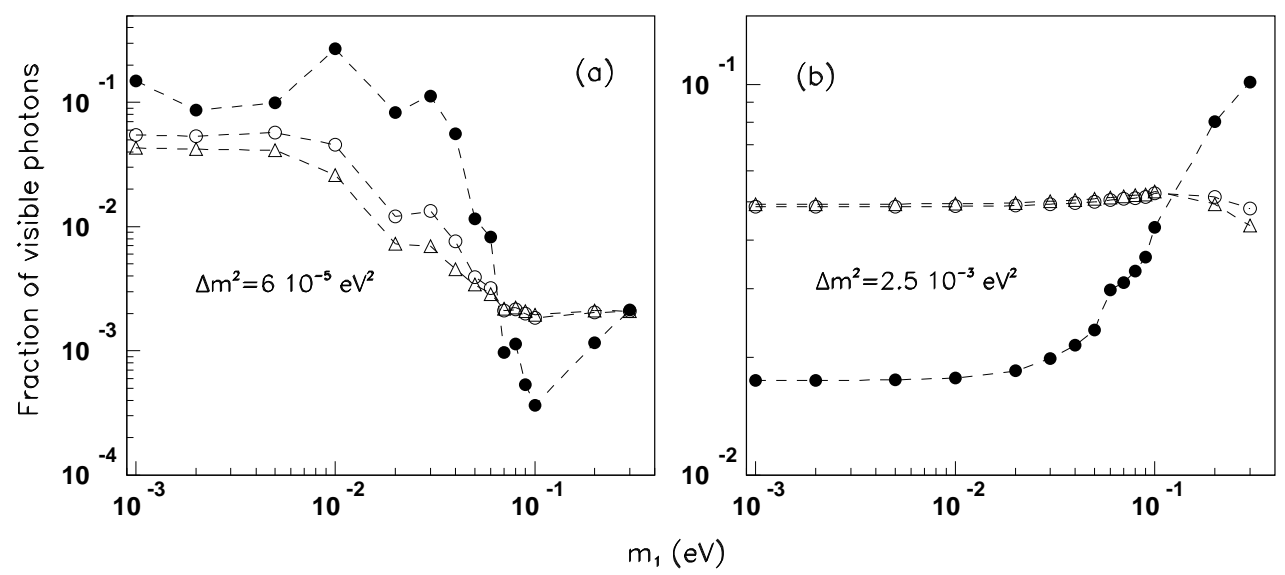

Fig. 5. The fraction of visible photons produced in the simulated radiative decays of massive solar neutrinos. The assumed squared mass differences are (a) $\Delta m^{2}=6 \times 10^{-5} \mathrm{eV}^{2}$ and (b) $2.5 \times 10^{-3} \mathrm{eV}^{2}$ (right). The light triangles, light circles and dark circles correspond to polarisations $\alpha=-1,0$ and +1 , respectively. The dashed lines are drawn only to guide the eye.

For each combination of neutrino mass and $\Delta m^{2}$ we requested $3 \times 10^{4}$ unweighted events yielding photons in the visible range. Assuming $\Delta m^{2}=$ $6 \times 10^{-5} \mathrm{eV}^{2}$ the total number of iterations was about $1.6 \times 10^{9}$, while for $\Delta m^{2}=2.5 \times 10^{-3} \mathrm{eV}^{2}$, about $3.3 \times 10^{7}$ generated events were needed. The simulated events were then weighted according to the integral of Eq. 3, as discussed in the previous Section. In weighting the events the factor $K$ in Eq. 3 was set to 1 , as it is a constant for each $\left(m_{2}, \Delta m^{2}\right)$ hypothesis. For this analysis we also imposed the initialization of the random number generator to be the same for each run.

Fig. 5 shows the fraction of visible photons, versus the mass of the lighter neutrino $\nu_{1}$. Fig. 5 a corresponds to $\Delta m^{2}=6 \times 10^{-5} \mathrm{eV}^{2}$, while the results for $\Delta m^{2}=2.5 \times 10^{-3} \mathrm{eV}^{2}$ are presented in Fig. $5 \mathrm{~b}$. The light triangles are obtained assuming $\alpha=1$, while the dark and light circles correspond to $\alpha=0$ and $\alpha=+1$, respectively.

As discussed in the previous Section, for each simulated neutrino decay event we numericaly integrated Eq. 3, thus obtaining a probability that includes the contributions from the kinematics of the decay itself as well as from the a priori request included in the simulation that the emitted photon reaches the detector. In Fig. 6 we present these probabilities, averaged over all Monte Carlo events yielding visible photons, versus the mass of the $\nu_{1}$ neutrino. Fig. 6a refers to $\Delta m^{2}=6 \times 10^{-5} \mathrm{eV}^{2}$, and Fig. $6 \mathrm{~b}$ to $\Delta m^{2}=2.5 \times 10^{-3} \mathrm{eV}^{2}$. The symbols used for different polarization states are the same as in Fig. 5. 


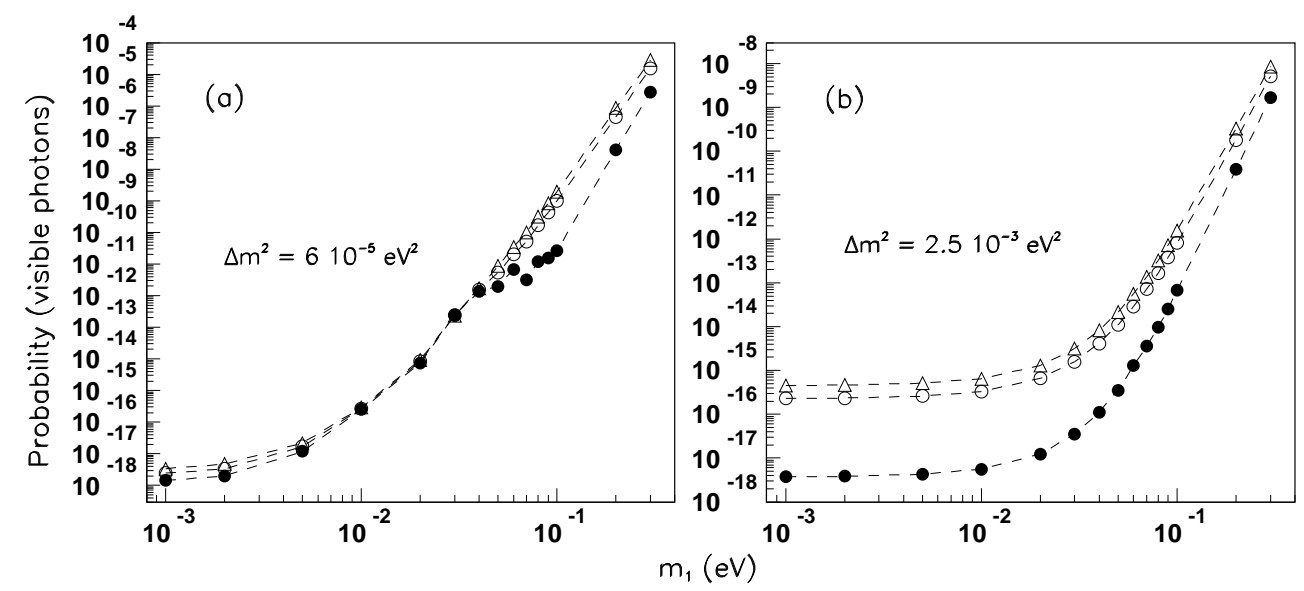

Fig. 6. Average probabilities for the neutrino radiative decay yielding visible photons inside the simulated analysis acceptance. The assumed squared mass differences are $\Delta m^{2}=6 \times 10^{-5} \mathrm{eV}^{2}$ (a) and $2.5 \times 10^{-3} \mathrm{eV}^{2}(\mathrm{~b})$. The light triangles, the light circles and the dark circles correspond to polarisations $\alpha=-1,0$ and +1 , respectively. The dashed lines are only meant to guide the eye.

The probabilities shown in Fig. 6 are very small, but one should consider that they apply to all the solar massive neutrinos that cross the "acceptance cone" of the detector between the Earth and the Moon (see Fig. 1).

Both Figs. 5 and 6 show a strongly non-linear behaviour, as the condition imposed to the photons to be in the visible spectrum selects different regions of the solar neutrino spectrum for different mass or polarization hypothesis. This effect is illustrated in Fig. 7, for three values of the neutrino mass $m_{1}$, $0.001,0.01$ and $0.1 \mathrm{eV}$ : the solid, dashed and dotted histograms respectively. Fig. 7a corresponds to $\Delta m^{2}=6 \times 10^{-5} \mathrm{eV}^{2}$, while Fig. $7 \mathrm{~b}$ to $\Delta m^{2}=2.5 \times 10^{-3}$ $\mathrm{eV}^{2}$. In all cases the polarization parameter $\alpha$ was assumed -1 .

Fig. 7 suggests that the high energy solar ${ }^{8} B$ neutrino tail does not yield visible photons through radiative decays. Such process could instead happen for low energy $p p$ neutrinos, with some contributions from the ${ }^{13} N,{ }^{15} \mathrm{O}$ neutrinos and from the ${ }^{7} \mathrm{Be}$ and pep lines; notice that these last contributions are more noticeable in Fig. 7a than in Fig. 7b.

In conducting an experiment searching for visible photons from a hypothetical radiative solar neutrino decay during TSE's and in choosing the appropriate data analysis methodology, the simulation of the expected signal is very important. Fig. 8 presents such simulations, in the same conditions and with the same conventions as in Fig. 7. The shape of the signals corresponding to different neutrino masses and mass differences (assuming left handed neutrinos, 


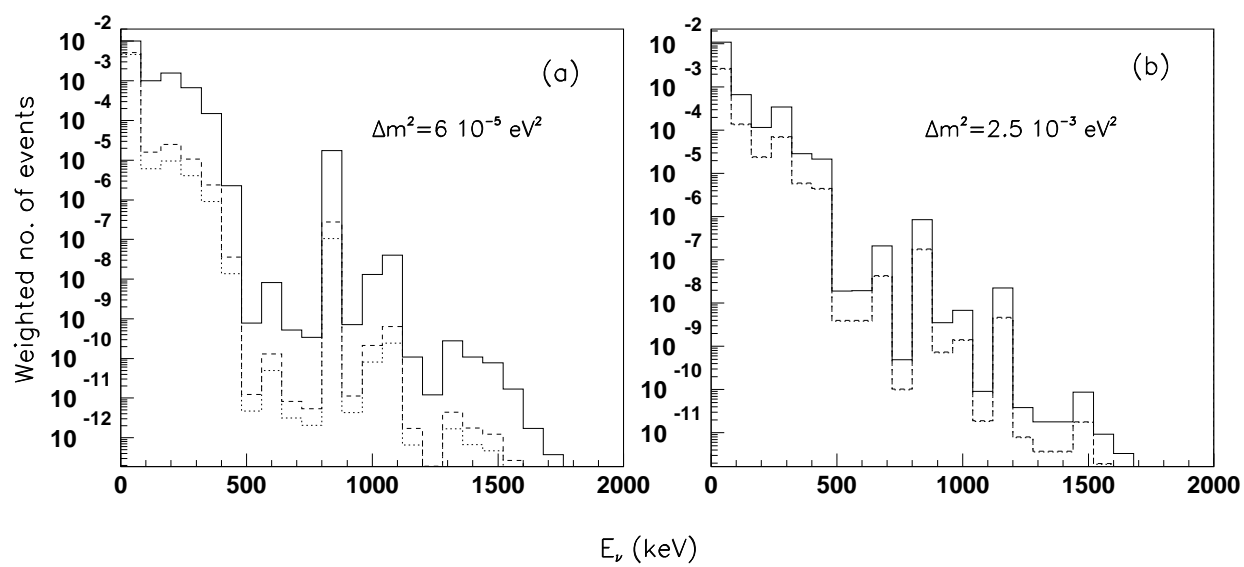

Fig. 7. The energy distribution of the solar neutrinos that yield visible photons through radiative decay, assuming $m_{1}=0.001 \mathrm{eV}$ (solid histograms), $0.01 \mathrm{eV}$ (dashed histograms) and $0.1 \mathrm{eV}$ (dotted histograms). The squared mass difference is assumed $6 \times 10^{-5} \mathrm{eV}^{2}$ (a) and $2.5 \times 10^{-3} \mathrm{eV}^{2}$ (b). In all cases $\alpha=-1$.

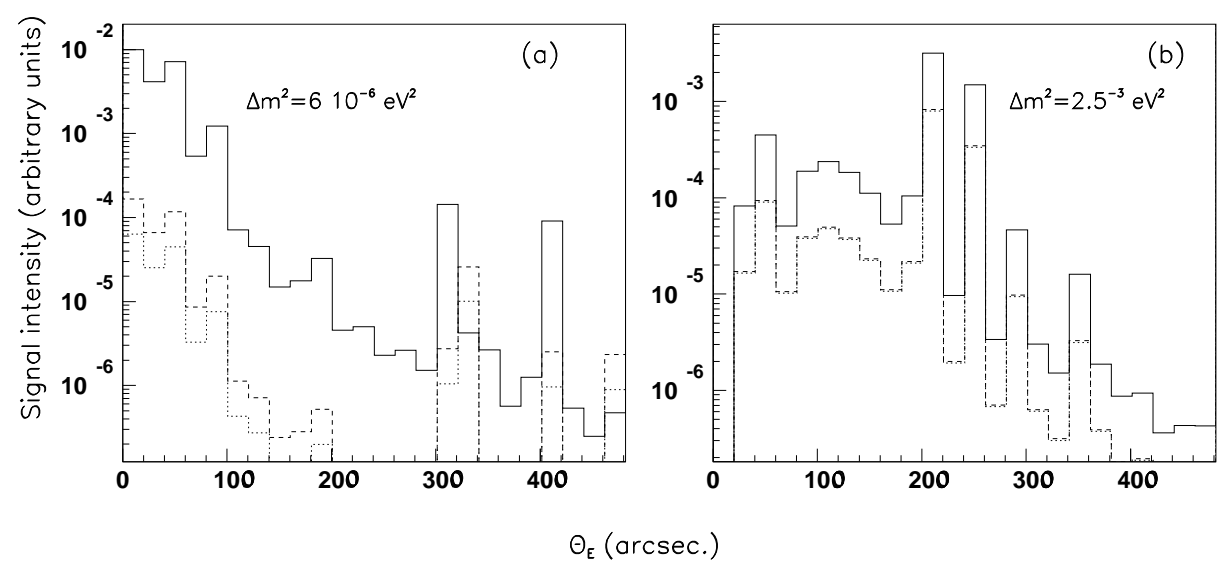

Fig. 8. The expected shapes of the visible signals produced by the hypothesized solar neutrino radiative decay, assuming $m_{1}=0.001 \mathrm{eV}$ (solid histograms), $0.01 \mathrm{eV}$ (dashed) histograms) and $0.1 \mathrm{eV}$ (dotted histograms). The squared mass difference is assumed to be $6 \times 10^{-5} \mathrm{eV}^{2}$ (a) and $2.5 \times 10^{-3} \mathrm{eV}^{2}$ (b). In all cases $\alpha=-1$.

thus $\alpha=-1$ ) is presented as the radial distribution of the "detected" luminosity. This is equivalent to the distribution of the weighted number of visible photons, averaged on circular corona around the same value of the incidence zenith angle $\theta_{E}$ (see Fig. 1).

The histograms in Fig. 8a correspond to the simulated $\nu_{2} \rightarrow \nu_{1}+\gamma$ decays. For all neutrino masses, the expected signal is concentrated at small $\theta_{E}$ angles 


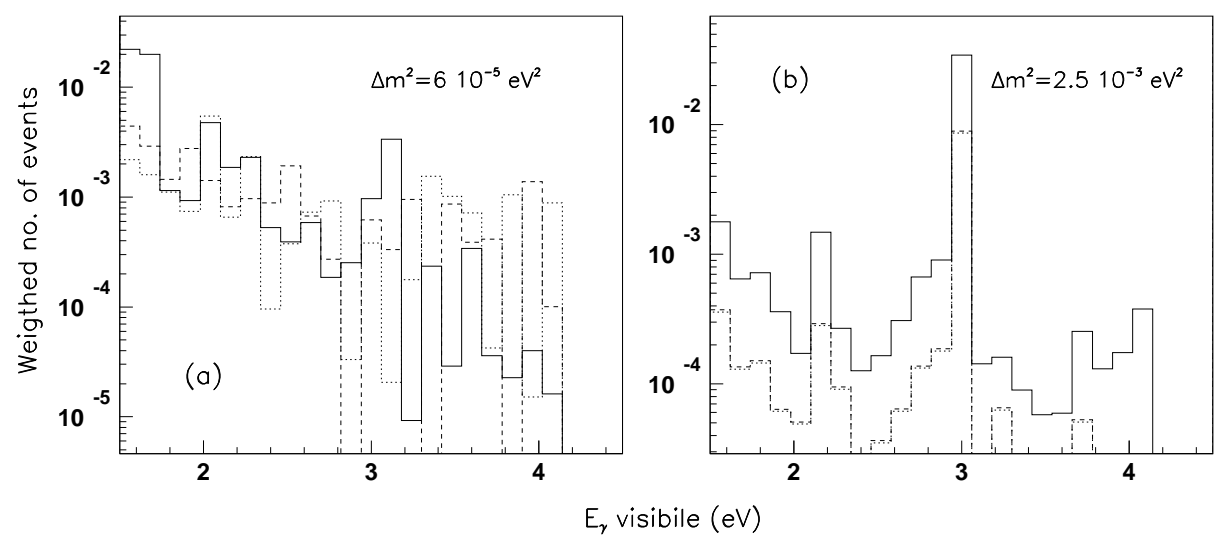

Fig. 9. The expected energy spectra of the visible signals produced by the hypothesized solar neutrino radiative decay, assuming $m_{1}=0.001 \mathrm{eV}$ (solid histograms), $0.01 \mathrm{eV}$ (dashed histograms) and $0.1 \mathrm{eV}$ (dotted histograms). The squared mass difference is assumed $6 \times 10^{-5} \mathrm{eV}^{2}$ (a) and $2.5 \times 10^{-3} \mathrm{eV}^{2}(\mathrm{~b})$. In all cases $\alpha=-1$.

(about 50 arcsec). The widths and shapes of the signals are sensitive to the mass assumed: the larger the mass, the narrower the signal band. The two peaks seen at about 300 and 400 arcsec. for $m_{1}=10^{-3} \mathrm{eV}$ could be correlated with the contribution of ${ }^{7} \mathrm{Be}$ or $p e p$ neutrinos; they are about a factor 100 lower than the central maxima, so their contribution is not measurable.

In the case of $\nu_{3} \rightarrow \nu_{1}+\gamma$ simulated decays (Fig. 8b), the signal is broader (about 250 arcsec) and is less sensitive to the mass choice. The peaks observed at about 250" could have a similar origin as those in Fig. 8a, but could also be statistical fluctuations.

If an experiment on solar neutrinos has also a good energy resolution, then further information could be obtained from the analysis of the spectra of the observed signal. Such spectra are shown in Fig. 9, assuming only left-handed neutrinos, and considering the same examples as in Figs. 7 and 8.

As for the shape of the signal, its spectral decomposition seems to be more sensitive to the neutrino mass values for the $\nu_{2} \rightarrow \nu_{1}+\gamma$ decays (Fig. 9a). In this case most of the visible photons are "detected" in the red part of the spectrum, while the spectra in Fig. 9b suggest a dominant signal in the green, due to the larger mass difference between $\nu_{3}$ and $\nu_{1}$.

Let us assume that an experiment as those simulated in this work would measure, during a TSE, an excess of visible photons $\Phi_{\text {obs. }}$ from the direction 
of the center of the sun, or, if the search yields a negative result, $\Phi_{\text {obs. }}$ is the photon flux of the observed fluctuations. In the first case, estimates of the neutrino lifetime could be done; otherwise, a lower experimental limit could be deduced. Assuming that solar (electron) neutrinos are superpositions of only two mass eigenstates,

$$
\left|\nu_{e}>=\right| \nu_{1}>\cos \theta+\mid \nu_{2}>\sin \theta
$$

where $m_{2}>m_{1}$ and $\theta$ is the mixing angle, the average lifetime (or its lower limit) $\tau$ of the $\nu_{2}$ neutrino could be computed from

$$
N_{\gamma}=P \Phi_{2} S_{M} t_{o b s}\left(1-e^{-\frac{<t_{M E}>}{\tau}}\right) e^{-\frac{t_{S M}}{\tau}}
$$

where $N_{\gamma}$ is the number of decay visible photons observed, $P$ are the mass - dependent probabilities shown in Fig. 6 a, $\Phi_{2}=\Phi_{\nu} \sin ^{2} \theta$, (where $\Phi_{\nu}$ is the flux of solar neutrinos at the Earth (or Moon) level), $S_{M}$ is the area of the Moon surface covered by the analysis (the base of the cone of angle $\theta_{E}$ in Fig. $1)$ and $t_{o b s}$ is the time of observation. $\left\langle t_{M E}\right\rangle$ is the average time spent by solar neutrinos inside the observation cone (about one third of the flight time from the Moon to the Earth), and $t_{S M}$ is the time of flight of the neutrinos from the Sun to the Moon. The low numerical values of the probabilities $P$ are compensated by the large solar neutrino flux, combined with the large area $S_{M}$, so an experiment as the one simulated here could yield at least significant upper limits on the $\nu_{2}$ lifetime $\tau$. In the conditions of a 3.5 minutes long TSE (as that of 2001) observed with an instrument with characteristics similar to those considered in this simulation, one would expect a $\nu_{2}$ lifetime (in the proper reference frame) sensitivity ranging from few seconds to about $10^{4}$ seconds, assuming $\nu_{2}$ neutrino masses of few $10^{-2} \mathrm{eV}$.

\section{Conclusions}

In this paper we presented a Monte Carlo simulation for an experiment looking for visible photons emitted by a possible solar neutrino radiative decay, during a total solar eclipse. It was shown that for neutrino masses smaller than $0.1 \mathrm{eV}$ and assuming squared mass differences in agreement with the Large Mixing Angle Solution (LMA) of the solar neutrino oscillations [15], such an experiment could give also an estimate of the neutrino mass.

The analysis of the experimental data collected by some of the authors during the 2001 TSE in Zambia is being completed using the simulation results obtained in this paper. 


\section{Acknowledgements}

We would like to aknowledge many colleagues for useful comments and discussions.

This work was founded by NATO Grant PST.CLG.977691 and partially supported by the Italian Space Agency (ASI) and the Romanian Space Agency (ROSA).

\section{References}

[1] G. Giacomelli and M. Sioli, Astroparticle Physics, hep-ex/0211035

[2] A.L. Melott, D.W. Sciama, Phys.Rev.Lett. 46 (1981) 1369-1372.

[3] D.W. Sciama, Nucl.Phys.Proc.Suppl. 38 (1995) 320-323.

[4] C. Birnbaun et al., Phys. Lett. B397 (1997) 143-146.

[5] J.-M. Frère and D. Monderen, Phys. Lett. B431 (1998) 368-373.

[6] S. Cecchini et al. (NOTTE Coll.), Astrophys. and Space Sci. 273 (2000) 35-41.

[7] S. Cecchini et al. (NOTTE Coll.), Limits on radiative decays of solar neutrinos from a measurement during a solar eclipse, hep-ex/0011048

[8] V. Popa et al. (NOTTE Coll.), Astrophys. and Space Sci. 282 (2002) 235-244.

[9] J.N. Bahcall, Standard Solar Models, astro-ph/9808162

[10] Q.R. Ahmad et al. (SNO Coll.), Phys. Rev. Lett. 87 (2001) 071301.

[11] Q.R. Ahmad et al. (SNO Coll.), Phys. Rev. Lett. 89 (2002) 011301.

[12] D.N. Spergel at al., First year Wilkinson Microwave Anisotropy Probe (WMAP) observations: Determination of cosmological parameters, Ap. J. (2003) accepted-

[13] M. Ambrosio et al. (MACRO Coll.), Phys. Lett. B434 (1998) 451; Phys. Lett. B357 (1995) 481.

[14] M. Ambrosio et al. (MACRO Coll.), Phys. Lett. B566 (2003) 35.

[15] Y. Fukuda et al. (Super-Kamiokande Coll.), Phys. Rev. Lett. 81 (1998) 1562; Nucl. Instrum. Meth. 503 (2003) 114.

[16] J.N. Bahcall, M.H. Pinsonneault and S. Basu, Ap. J. 555 (2001) 990-1012.

[17] http://www.sns.ias.edu/ jnb/

[18] K. Hagiwara et al. (Particle Data Group), Phys. Rev. D66 (2002) 010001. 